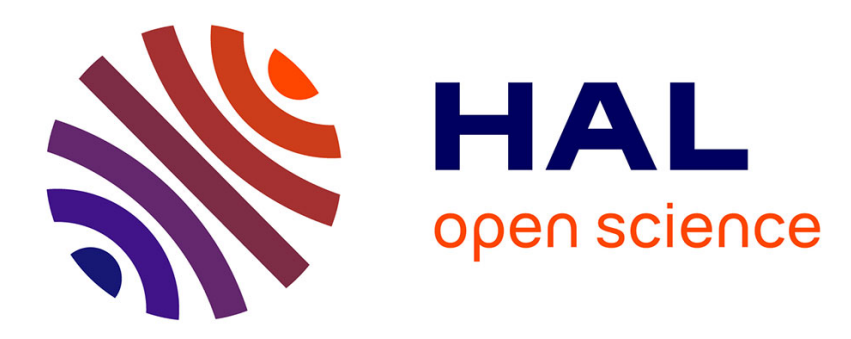

\title{
Étude magnétostatique de l'aimantation nucléaire des protons de l'eau
}

\author{
M. François, J.P. Pozzi
}

\section{To cite this version:}

M. François, J.P. Pozzi. Étude magnétostatique de l'aimantation nucléaire des protons de l'eau. Revue de Physique Appliquée, 1970, 5 (2), pp.309-316. 10.1051/rphysap:0197000502030900 . jpa-00243393

\section{HAL Id: jpa-00243393 https://hal.science/jpa-00243393}

Submitted on 1 Jan 1970

HAL is a multi-disciplinary open access archive for the deposit and dissemination of scientific research documents, whether they are published or not. The documents may come from teaching and research institutions in France or abroad, or from public or private research centers.
L'archive ouverte pluridisciplinaire HAL, est destinée au dépôt et à la diffusion de documents scientifiques de niveau recherche, publiés ou non, émanant des établissements d'enseignement et de recherche français ou étrangers, des laboratoires publics ou privés. 


\title{
ÉTUDE MAGNÉTOSTATIQUE DE L'AIMANTATION NUCLÉAIRE DES PROTONS DE L'EAU
}

\author{
M. FRANÇOIS et J. P. POZZI \\ Laboratoire de Mécanique des Fluides, Faculté d'Orsay, 91 \\ Laboratoire de Géomagnétisme, 4, avenue de Neptune, 94, Saint-Maur-des-Fossés
}

(Reçu le 24 juin 1969, revisé le 17 novembre 1969)

\begin{abstract}
Résumé. - En utilisant la méthode de prépolarisation et retournement par passage adiabatique rapide, nous avons effectué une mesure statique de l'aimantation nucléaire de l'eau et déduit le temps de relaxation spin-réseau $T_{1}$ ainsi qu'une valeur de la susceptibilité nucléaire $\chi_{0}$. Nous faisons une description détaillée du dispositif utilisé en insistant particulièrement sur les conditions expérimentales nécessaires à l'obtention d'une bonne précision. La valeur de $\chi_{0}$ trouvée est $\chi_{0}=3,22 \pm 0,1 \times 10^{-10}$ dans le système u. e. m. c. g. s.
\end{abstract}

Abstract. - Using the method of prepolarization and fast adiabatic passage, we have made a static measurement of the nuclear magnetization of the protons of water from which we have decuced the spin-lattice relaxation time $T_{1}$ and a value for the nuclear susceptibility $\chi_{0}$. The experimental design is described in detail, with particular attention to the experimental conditions necessary to achieve high precision. The obtained value of $\chi_{0}$ is $3.22 \pm 0,1 \times 10^{-10}$ in the e. $\mathrm{m}$. $\mathrm{u}$. c. g. s. system.

Introduction. - Les méthodes d'observation du magnétisme nucléaire sont habituellement divisées en deux catégories: les méthodes par résonance et les méthodes statiques.

D'une façon générale, pour determiner la différence d'énergie $\Delta E$ entre 2 niveaux d'un système matériel, une méthode statique consistera à trouver une grandeur mesurable $M$ en fonction de $\Delta E$ et à la mesurer. La précision, qui est au plus celle de la mesure de $M$, est souvent médiocre, d'autant plus que la relation fonctionnelle entre $\Delta E$ et $M$ peut contenir d'autres paramètres dont la connaissance est également requise, ce qui n'est pas le cas dans les méthodes résonnantes qui se ramènent à des mesures de fréquence. Ainsi, s'il est naturel de songer à une mesure statique du paramagnétisme nucléaire, l'extrême petitesse $\mu_{N}$ (moment magnétique nucléaire) explique la difficulté et le très petit nombre de mesures statiques effectuées à ce jour.

Néanmoins, en 1937, Lazarew et Schubnikow [1], utilisant une première méthode statique, réussissaient à mesurer les forces subies par un échantillon macroscopique dans un champ inhomogène. L'échantillon était de l'hydrogène solide à très basse température et ces auteurs ont pu déduire une valeur approchée de la susceptibilité paramagnétique nucléaire. Cette technique a été reprise par Evans [2] à température ordinaire avec un échantillon d'hexaéthylbenzène. Evans a pu mettre en évidence la variation de la force subie par l'échantillon lors d'une irradiation à la fréquence de résonance.
Poulis a repris cette expérience et a pu arriver à une bonne mesure de la susceptibilité magnétique nucléaire des protons :

$$
\chi_{0}=3,23 \times 10^{-10}
$$

dans le système u. e. m. c. g. s.

Par ailleurs, en 1951, A. Kastler suggère la possibilité d'étudier des aimantations nucléaires de longue persistance sur des corps solides par une deuxième méthode statique qui consiste à mesurer le champ produit par l'échantillon. La mise au point récente de magnétomètres astatiques de très haute sensibilité a permis de mettre en pratique cette idée que nous décrivons ici.

En utilisant des substances très pures où le temps de relaxation nucléaire est long, on peut envisager l'étude du phénomène en dehors du champ inducteur : lorsqu'on éloigne l'échantillon nucléaire "polarisé » du champ, il conserve pendant un certain temps son aimantation qui ne décroît que lentement. On peut dire que les noyaux, en quittant le champ, subissent une désaimantation adiabatique qui les porte à une température de spin nucléaire extrêmement faible et qu'ils ne se réchauffent que lentement au contact du réseau.

L'expérience a été réalisée sur de l'eau en circulation dans un circuit fermé, ce qui résout le problème du transport de l'échantillon hors du champ inducteur jusqu'au magnétomètre.

Pour révéler le paramagnétisme nucléaire, nous avons utilisé, d'une part la méthode de polarisation 
préalable en faisant passer le liquide dans l'entrefer d'un électro-aimant, et d'autre part une méthode d'inversion $\mathrm{du}$ sens de l'aimantation par passage adiabatique rapide.

Les variations du champ magnétique qui, au voisinage de l'échantillon de mesure, résultant de cette inversion, sont décelées par le magnétomètre.

I. Polarisation préalable. - 1. Particules dans un Champ magnétique. Polarisation. - Considérons un noyau qui possède un moment magnétique $\mu$ lié à son spin. Dans le cas des protons le nombre de spin est $I=\frac{1}{2}$. A l'équilibre thermodynamique que, pour simplifier, nous supposerons atteint, le nombre des noyaux orientés selon le champ $H: N(+\mu)$ est supérieur au nombre des noyaux orientés en sens inverse $N(-\mu)$ et :

$$
\frac{N(+\mu)}{N(-\mu)}=\exp \left(\frac{2 \mu H}{k T}\right)
$$

le volume de substance possède alors une aimantation nucléaire $M$, orientée selon le champ appliqué $H$, qui a pour valeur : $M=\mu[N(+\mu)-N(-\mu)]$. Si $N_{0}$ est le nombre de particules par $\mathrm{cm}^{3}$ on peut écrire, considérant que

$$
\frac{2 \mu H}{k T} \ll 1: M=\frac{\mu^{2} N_{0} H}{k T} .
$$

Le coefficient de proportionnalité entre $M$ et $H$ est appelé susceptibilité nucléaire statique $\chi_{0}$ de la substance et :

$$
\chi_{0}=\frac{\mu^{2} N_{0}}{k T} .
$$

Dans le cas général, si le spin nucléaire a pour valeur $I$ on a :

$$
M=\frac{N_{0}(\gamma h)^{2}}{3 k T} I(I+1) H
$$

où $\gamma$ est le rapport gyromagnétique du noyau considéré.

Toute modification dans l'aimantation, caractéristique de la réorientation de noyaux par échange d'énergie avec le milieu, s'effectue avec une constante de temps $T_{1}$ appelé temps de relaxation spin-milieu.

Ainsi, un système de noyaux préalablement «polarisé » dans un champ intense, puis porté dans un champ faible $H_{0}$ voit son aimantation évoluer suivant la loi :

$$
\frac{\mathrm{d} M}{\mathrm{~d} t}=\frac{\chi_{0} H_{0}-M}{T_{1}}
$$

Les propriétés adiabatiques de l'aimantation lui imposent de rester alignée avec $H_{0}$ lorsque les variations de ce dernier sont suffisamment lentes.

2. CAS DES Liquides EN ÉCOULEMENT. RENDEMENT DE LA POLARISATION. - Soient $t_{\mathrm{r}}$ le temps de polarisation du liquide dans l'électro-aimant; $t_{\mathrm{c}}$ le temps de dépolarisation lors du transport du liquide jusqu'à la cellule de mesure, $H_{\mathrm{p}}$ le champ de polarisation, $H_{0}$ le champ environnant.

Après passage dans l'électro-aimant, le liquide possède une aimantation qui s'écrit :

$$
M_{1}=\chi_{0} H_{\mathrm{p}}\left[1-\exp \left(\frac{-t_{\mathrm{r}}}{T_{1}}\right)\right]+\chi_{0} H_{0} \exp \left(\frac{-t_{\mathrm{r}}}{T_{1}}\right)
$$

soit au niveau de la cellule de mesure :

$$
\begin{array}{r}
M_{0}=\chi_{0} H_{0}\left[1-\exp \left(\frac{-t_{\mathrm{c}}}{T_{1}}\right)\right]+M_{1} \exp \left(\frac{-t_{\mathrm{c}}}{T_{1}}\right) \\
M_{0}=\chi_{0} H_{0}\left[1-\exp \left(\frac{-t_{\mathrm{c}}}{T_{1}}\right)\right]+\chi_{0} H_{0} \exp \left(-\frac{t_{\mathrm{r}}+t_{\mathrm{c}}}{T_{1}}\right) \\
+\chi_{0} H_{p}\left[1-\exp \left(\frac{-t_{\mathrm{r}}}{T_{1}}\right)\right] \exp \left(\frac{-t_{\mathrm{c}}}{T_{1}}\right)
\end{array}
$$

et dans les conditions expérimentales où $H_{0}$ est très inférieur à $H_{\mathrm{p}}\left(H_{\mathrm{p}} / H_{0} \simeq 10^{4}\right)$, l'équation devient :

$$
M_{0}=\chi_{0} H_{\mathrm{p}}\left[1-\exp \left(\frac{-t_{\mathrm{r}}}{T_{1}}\right)\right] \exp \left(\frac{-t_{\mathrm{c}}}{T_{1}}\right) \text {. }
$$

On peut alors définir un « rendement» de la polarisation comme le rapport de l'aimantation restant au niveau de la cellule de mesure à celle qui serait acquise dans l'électro-aimant à l'équilibre thermodynamique, soit :

$$
y=\left[1-\exp \left(\frac{-t_{\mathrm{r}}}{T_{1}}\right)\right] \exp \left(\frac{-t_{\mathrm{c}}}{T_{1}}\right)
$$

$\chi_{0}$ a donc pour expression

$$
\chi_{0}=\frac{M_{0}}{H_{\mathrm{p}} y} .
$$

En pratique, soit $V_{\mathrm{r}}$ et $V_{\mathrm{c}}$ les volumes balayés par le liquide dans le réservoir de polarisation, et la canalisation et $d$ le débit ; si l'on peut considérer que les particules fluides ont un temps de séjour homogène dans $V_{\mathrm{r}}$ et dans $V_{\mathrm{c}}$ (nous examinerons plus loin ce problème) l'expression (1) devient :

$$
\begin{aligned}
y & =\exp \left(\frac{-V_{\mathrm{c}}}{T_{1} d}\right)\left[1-\exp \left(\frac{-V_{\mathrm{r}}}{T_{1} d}\right)\right] \\
& =\left[\exp \left(\frac{-\alpha V_{\mathrm{r}}}{T_{1} d}\right)\right]\left[1-\exp \left(\frac{-V_{\mathrm{r}}}{T_{1} d}\right)\right]
\end{aligned}
$$

avec $\alpha=V_{\mathrm{c}} / V_{\mathrm{r}}$. L'expression (2) admet un maximum pour une valeur $d_{\mathbf{M}}$ du débit. Par ailleurs, le calcul montre que $\mathrm{d} y_{\mathrm{M}} / \mathrm{d} T_{1}=0$.

II. Inversion de l'aimantation nucléaire par passage adiabatique rapide. - L'inversion des populations des niveaux d'énergie $N(+\mu), N(-\mu)$, ou, du point de vue macroscopique, l'inversion de l'aimantation nucléaire, qui s'oriente alors à l'opposé du champ directeur, est réalisée par le procédé du «passage adiabatique rapide » proposé par Bloch [4] lors de la découverte de la R. M. N. et étudié et mis au point 
par H. Benoit [5] suivant une méthode simple que nous avons utilisée : le liquide coule dans une région où règne un champ radiofréquence alternatif d'amplitude $2 H_{1}$ et de pulsation $\omega_{1}$ en présence d'un champ statique $H_{0}$ inhomogène. La valeur de ce champ est telle que le liquide traverse une zone de résonance où l'on a $\omega_{1}=\gamma H_{0}$. Les spins sont retournés si

$$
\gamma H_{1}^{2} \gg \mathrm{d} H_{0} / \mathrm{d} t
$$

où $\mathrm{d} H_{0} / \mathrm{d} t$ est la vitesse de variation du champ $H_{0}$ lorsqu'on se déplace avec un élément du liquide. $\mathbf{H}$. Benoit et $\mathbf{M}$. Henon ont étudié la variation du facteur de retournement en fonction de :

$$
a=\frac{\gamma H_{1}^{2}}{\mathrm{~d} H_{0} / \mathrm{d} t} \text { variant de } \frac{1}{30} \text { à } 10 \text {. }
$$

Le retournement est pratiquement parfait pour $a=3$. Les caractéristiques de l'appareillage utilisé nous permettent d'affirmer que cette condition est remplie. On contrôle à chaque instant la qualité du retournement par un dispositif de Résonance Nucléaire classique.

III. Magnétomètre astatique. - L'appareil utilisé pour mesurer la variation de champ magnétique produite au voisinage de l'échantillon par le retournement de l'aimantation nucléaire est un magnétomètre astatique employé au Laboratoire de Géomagnétisme pour les mesures d'aimantations rémanentes de roches. Cet appareil, décrit par E. Thellier (1938) [6] et perfectionné récemment (J. P. Pozzi et E. Thellier, 1961) [7] et J. P. Pozzi (1967) [8], permet de mesurer des aimantations extrêmement faibles de l'ordre de $10^{-7}$ à $10^{-8} /$ u. e. m. c. g. s. Le magnétomètre utilisé est formé de trois aimants sphériques en platine-cobalt enfilés et collés à égale distance les uns des autres sur une fine tige de silice soigneusement rectifiée. Les moments magnétiques $M$ des deux aimants situés aux extrémités de la tige sont équipollents. L'aimant central a un moment magnétique $2 M$ et leur est antiparallèle. L'ensemble, qui pèse moins d'un gramme, est suspendu à un fil de silice de $5 \mu$ dans une enceinte vidée. Les déviations sont lues à l'aide d'un miroir. Le moment magnétique total du système d'aimants est très sensiblement nul (en réalité il est de $2 \mathrm{M} / 5000$ ) et le magnétomètre est pratiquement insensible à des perturbations se manifestant sur lui par un champ uniforme. De plus, sa symétrie le rend également insensible aux perturbations présentant un gradient constant sur la hauteur du système d'aimants.

IV. Dispositif expérimental. - 1 . Description. Une pompe centrifuge à corps en acier inoxydable (Fig. 1) assure la circulation de l'eau. La pompe aspire le liquide dans un réservoir d'une capacité de 401 (2). Un ensemble de débitmètres (3) mesure avec une grande précision les débits d que l'on ajuste par une dérivation comportant une vanne à opercule (4) entre $3 \mathrm{~cm}^{3} / \mathrm{s}$ et $100 \mathrm{~cm}^{3} / \mathrm{s}$. L'eau est ensuite envoyée dans

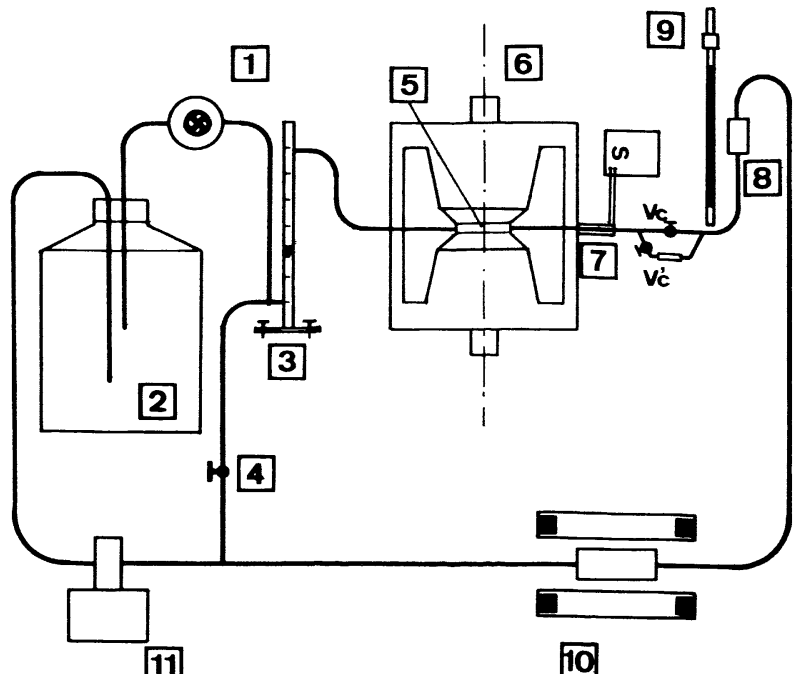

Fig. 1. - Dispositif expérimental.

un réservoir appelé réservoir de polarisation (5) de forme extérieure cylindrique placé dans l'entrefer d'un électro-aimant (6) dont le champ agit parallèlement à l'axe du cylindre.

Après passage dans le dispositif de retournement (7), une canalisation de petit volume en verre, conduit rapidement l'eau polarisée dans une cellule de mesure (8) (également en verre), située à proximité du magnétomètre (9) puis dans un dispositif de résonance magnétique nucléaire classique (10) d'où elle est renvoyée au réservoir. Par un jeu de robinets le volume $V_{\mathrm{c}}$ de la canalisation peut être augmenté d'une quantité connue $V_{\mathrm{c}}^{\prime}$.

Les déviations $S$ enregistrées au magnétomètre pour différents débits sont comparées aux valeurs théoriques de l'équation (2) :

$$
y_{\alpha, T_{1}}=y(\mathrm{~d})
$$

de cette comparaison on tire $\alpha, T_{1}$ et $y_{\mathrm{M}}$.

2. Précautions expérimentales. - a) Pureté $d u$ liquide. - Il est nécessaire d'utiliser de l'eau de caractéristiques physiques constantes et connues ; l'eau est spécialement permutée et distillée et, pour éviter toute dissolution de gaz, par exemple d'oxygène qui influence très fortement $T_{1}$, les manipulations se font sous pression d'azote pur (de teneur en $\mathrm{O}_{2}<10^{-5}$ ) dans le circuit et les récipients. Une cellule de mesure de résistivité montée sur le circuit permet de contrôler à chaque instant la valeur de cette caractéristique physique.

b) Stabilisation de température. - Les considérations qui suivent, montrent qu'il est indispensable de stabiliser la température du liquide.

- La susceptibilité magnétique nucléaire varie proportionnellement à $1 / T$.

- L'expression du temps de relaxation longitudinal $T_{1}$ peut s'écrire, en fonction de la température : 
$T_{1}=A T / \eta$ où $A$ est une constante et $\eta$ la viscosité de l'eau. Entre $15^{\circ} \mathrm{C}$ et $19^{\circ} \mathrm{C}$ la variation de $\eta$ est approximativement telle que $\Delta \eta / \eta=0,0253 /{ }^{\circ} \mathrm{C}$; le terme $\Delta \eta / \eta$ est prépondérant dans $\Delta T_{1} / T_{1}$.

- Nous verrons dans l'interprétation des résultats que le rapport $V_{\mathrm{c}} / V_{\mathrm{r}}=\alpha$ joue un très grand rôle.

Ce rapport dépend de façon complexe des conditions hydrodynamiques d'écoulement et, de ce fait, de la température par l'intermédiaire de la viscosité. La stabilisation à $0,2^{\circ} \mathrm{C}$ autour de $19^{\circ} \mathrm{C}$ que nous avons adoptée rend négligeable, comme nous le verrons, l'influence des variations de température par rapport aux autres causes d'incertitude.

c) Environnement magnétique. — Les distances entre les différents appareils doivent être aussi petites que possible, de façon à conserver un polarisation acceptable au niveau des éléments de mesure. Le champ de fuite est l'électro-aimant, bien que réduit au maximum par construction, est très grand par rapport aux champs mesurés au niveau des aimants du magnétomètre (dans la configuration adoptée pour les mesures, il est à peu près $10^{4}$ fois plus grand).

Deux bobines de Helmholtz, l'une d'axe horizontal, l'autre d'axe vertical, permettent de compenser la composante horizontale du champ magnétique terrestre et de faire varier l'intensité du champ vertical appliqué sur la cellule de mesure. Pendant son transport, de l'électro-aimant au magnétomètre, le liquide doit nécessairement être soumis à un champ magnétique directeur $H_{0}$ qui résulte ici de la composition des champs de fuite de l'électro-aimant des champs produits par les bobines de Helmholtz et du champ terrestre. Les propriétés adiabatiques de l'aimantation imposent l'étude de l'environnement magnétique le long du parcours du liquide polarisé. Pour illustrer ces remarques, on a représenté (Fig. 2) les variations

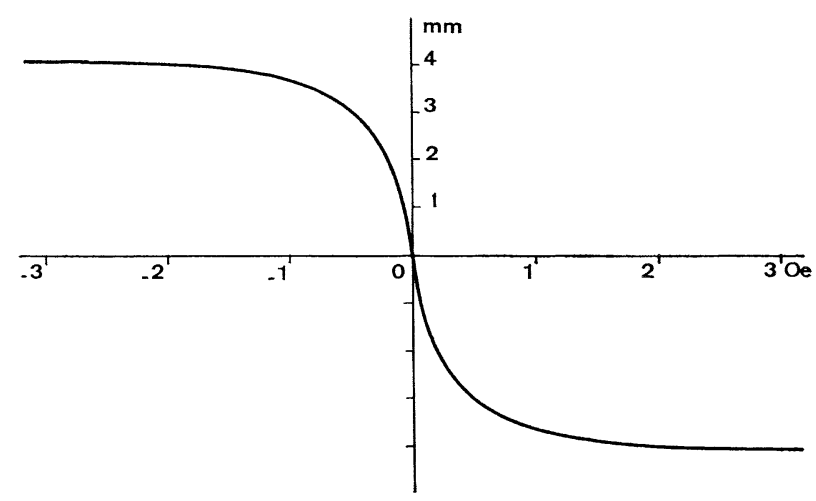

FIG. 2. - Variation du signal observé (déviation du magnétomètre) en fonction de l'intensité du champ directeur vertical appliqué sur la cellule de mesure.

de l'intensité du signal (déviations du magnétomètre), en fonction du champ vertical régnant au niveau de la cellule de mesure et (Fig. 3) le champ directeur $H_{0}$ dans les conditions d'expériences.

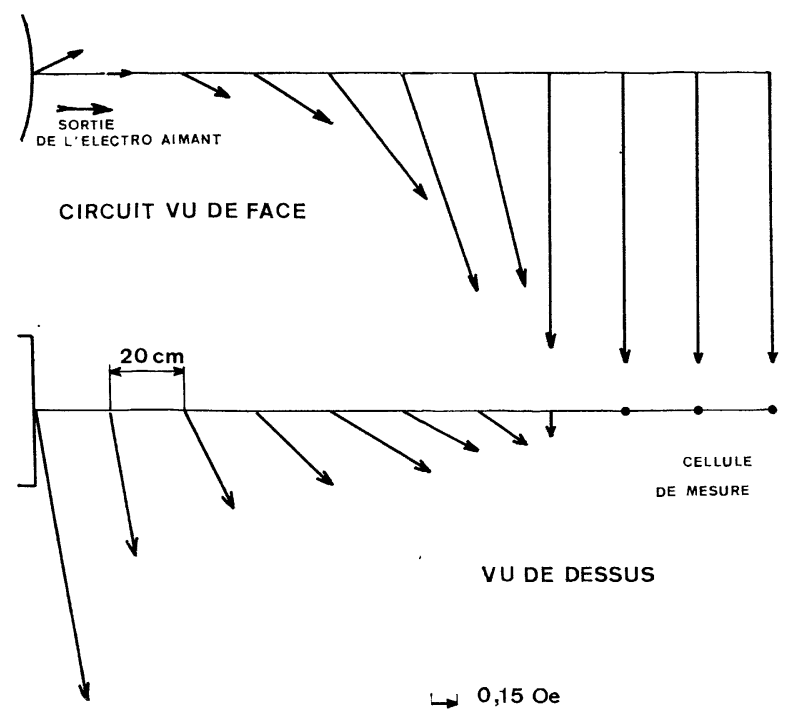

FIG. 3. - Intensité et direction du champ directeur qui règne sur le parcours du liquide polarisé.

V. Conditions hydrodynamiques d'écoulement. Comme nous l'avons vu (I.2), si les temps de séjour sont homogènes, l'expression (1) devient :

$$
y=\exp \left(\frac{-\alpha V_{\mathrm{r}}}{T_{1} d}\right)\left[1-\exp \left(\frac{-V_{\mathrm{r}}}{T_{1} d}\right)\right]
$$

(2) est approchée à $10^{-4}$ environ puisque la polarisation nucléaire acquise dans le champ terrestre $\chi_{0} H_{0}$ a été négligée devant $\chi_{0} H_{\mathrm{p}}$. Or, la méthode utilisée pour la détermination de $\alpha$ et $T_{1}(\mathrm{IV}, 1)$ implique que $V_{\mathbf{r}}$ et $V_{\mathrm{c}}$ soient indépendants du débit, et que l'on puisse définir les temps de séjour $t_{\mathrm{r}}$ et $t_{\mathrm{c}}$; au point de vue hydrodynamique cette condition revient à obtenir, outre des temps de séjour homogènes pour chaque débit, un régime d'écoulement indépendant du débit.

Dans le réservoir de polarisation, on peut définir dans ces conditions un temps $t_{\mathbf{r}}$ moyen égal à $V_{\mathbf{r} / \mathbf{d}}$.

Dans la cellule de mesure, le problème est différent ; en effet, comme on le verra, la méthode de correction d'étalonnage tient compte de la relaxation de l'aimantation nucléaire le long de cet échantillon, quelle que soit sa forme, à condition qu'il n'y ait pas de zones mortes.

Il est, d'autre part, nécessaire que l'écoulement soit turbulent en tout point du circuit. Dans ce cas, les variations de la couche limite avec le débit sont pratiquement sans influence sur $V_{\mathrm{c}}$ et $V_{\mathrm{r}}$. Par ailleurs, si la position et la vitesse d'une particule changent constamment en fonction du temps, statistiquement, la répartition des positions et des vitesses est uniforme pour un élément de volume donné ( $t_{\mathrm{r}}$ et $t_{\mathrm{c}}$ ont alors un sens). Remarquons qu'en régime laminaire ces propriétés ne sont plus vérifiées et il faudrait, en toute rigueur, s'intéresser à l'histoire des particules fluides. Bien qu'il soit difficile d'affirmer qu'à un débit donné le régime est rigoureusement laminaire dans tout le circuit, étant donné sa configuration, l'expérience 
montre que pour de faibles nombres de Reynolds (inférieurs à 500), l'expression donnant le rendement théorique n'est plus vérifiée avec la même précision. Ces considérations prendraient toute leur importance lorsqu'on étudie des substances à long temps de relaxation, ex. : Benzène $\left(T_{1}=20 \mathrm{~s}\right)$.

Toutes ces conditions étant remplies, à un régime donné les temps $t_{\mathrm{r}}$ et $t_{\mathrm{c}}$ subissent des fluctuations en raison des petites instabilités du régime. Ces fluctuations se traduisent par des variations de l'aimantation nucléaire mesurée de valeur moyenne non nulle et nous semblent être responsables d'une part importante du bruit de signal du magnétomètre.

Nous nous sommes attachés dans un premier temps à résoudre le problème de l'écoulement dans la cellule de mesure. Une solution simple consiste à utiliser une cellule de très petites dimensions, par exemple $3,5 \mathrm{~cm}^{3}$, comme cela avait été fait dans l'expérience citée en référence [8] ; mais dans ce cas les déviations enregistrées au magnétomètre sont petites et la précision relative médiocre. Nous avons donc étudié une cellule (Fig. 4) de plus grand volume comprenant une arrivée de liquide tangentielle et une sortie axiale, les dimensions sont adaptées à la géométrie du système d'aimants du magnétomètre. Une tige centrale fixe l'axe du tourbillon et donne un écoulement tourbillonnaire parfaitement stable contrôlé au préalable par visualisation en utilisant des particules d'aluminium en suspension ; la suppression de la partie centrale de l'échantillon ne fait perdre que $8 \%$ du signal par rapport à une cellule complète.

D'autre part, il est intéressant d'avoir un réservoir de volume $V_{\mathbf{r}}$ aussi grand que possible, sans zones

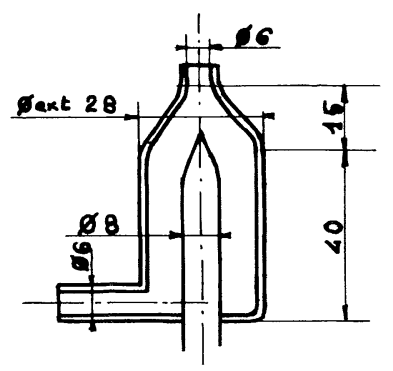

A

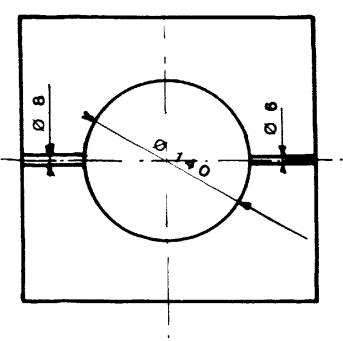

B

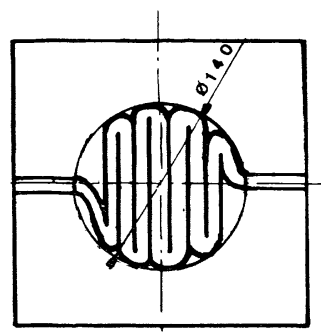

C
Fig. 4. - 4a) Cellule de mesure. 4b) Réservoir de polarisation du type cylindrique. $4 c$ ) Réservoir de polarisation utilisé pour les mesures. mortes et assurant un temps de séjour homogène ; il est normal de songer à utiliser tout le volume de l'entrefer par un réservoir cylindrique (Fig. 4b).

Une étude hydrodynamique de l'écoulement dans un tel réservoir montre hélas l'existence de 2 zones mortes symétriques dont le volume est fonction du débit. L'instabilité de l'écoulement est acceptable puisqu'elle se traduit par un bruit signal de l'ordre de $1,5 \%$ du signal. Cependant, $V_{\mathrm{r}}$ n'est pas rigoureusement constant en fonction du débit, ce qui rend imprécis la détermination de $\alpha$ et $T_{1}$. La précision globale est de l'ordre de $6 \%$. On peut penser par ailleurs, pour conserver un grand $V_{\mathrm{r}}$, disposer un système de diffuseur situé hors des pièces polaires; l'écoulement, vérifié par visualisation, est, dans ce cas, beaucoup plus homogène et $V_{\mathrm{r}}$ pratiquement constant mais la stabilité du signal moins bonne $(3 \%$ de bruit de signal, ce qui montre que $t_{\mathrm{r}}$ n'est pas homogène). Le réservoir que nous avons utilisé (Fig. $4 c$ ), le seul qui ait donné entière satisfaction, est formé d'un tube replié sur lui-même dans tout l'entrefer : dans ce cas $V_{\mathrm{r}}$ est parfaitement défini et la stabilité de l'écoulement excellente.

Le circuit utilisé est donc formé de ce dernier réservoir associé à la cellule de mesure décrite. Avec ce circuit, il est possible de faire coïncider, comme nous le verrons dans les résultats, avec une grande précision, la courbe expérimentale donnant la polarisation en fonction du débit $y\left(V_{\mathrm{r}}, \alpha, T_{1}\right)$ et de déterminer $V_{\mathrm{r}}, \alpha$, $T_{1}$, ce qui n'était pas possible avec des circuits composés d'autres éléments. Les seuls écarts constatés sont dans les très bas débits où probablement le régime devient laminaire dans certaines portions du circuit, en particulier dans le réservoir où le nombre de Reynolds tombe à 250 pour un débit de $3 \mathrm{~cm}^{3} / \mathrm{s}$.

En conclusion, si l'on se contente d'une précision de $6 \%$ environ sur $y_{M}$ il n'est pas nécessaire d'étudier en détail les conditions d'écoulement du liquide polarisé. Mais dans le cadre des précautions expérimentales mises en œuvre $(I V, 2)$ une telle étude est indispensable. Son extrême complexité impose pratiquement le choix de configurations très simples où l'on puisse effectivement contrôler les conditions d'écoulement.

VI. Résultats expérimentaux. - 1. ETAlONNAGE. - En général, les mesures magnétométriques sont faites par comparaison directe du moment magnétique mesuré avec celui de bobines étalons. En ce qui concerne l'expérience que nous décrivons il ne nous a pas été possible de procéder ainsi. En effet, l'étude hydrodynamique de l'écoulement dans la cellule de mesure nous a amenés à utiliser des cellules de formes compliquées.

Dans le cas de cellule de mesure de très faible dimension $\left(3,5 \mathrm{~cm}^{3}\right)$ une méthode simple consiste à comparer l'aimantation nucléaire à l'aimantation diamagnétique acquise par de l'eau pure placée dans la cellule de mesure et soumise à un champ connu.

Pour des cellules de dimensions plus importantes 
(par exemple $25 \mathrm{~cm}^{3}$ ) cet étalonnage n'est qu'approché puisque l'on compare une aimantation uniforme à une aimantation qui décroît sur la hauteur de la cellule $\mathrm{du}$ fait de la relaxation nucléaire ; il faut alors lui apporter une légère correction.

2. Correction D'ÉTAlonnage. - Le facteur correctif est essentiellement fonction du débit et des conditions hydrodynamiques d'écoulement. Nous avons imaginé la méthode suivante de correction d'étalonnage. Au cours de l'étalonnage par le diamagnétisme nous cherchons, en déplaçant la cellule de mesure parallèlement à son axe, pour quelle position la déviation obtenue est nulle. Nous divisons ainsi la cellule en 2 zones A et B situées respectivement audessous et au-dessus du niveau de l'aimant central où elles produisent des champs égaux et opposés.

Rétablissons l'écoulement du liquide polarisé ; si les deux zones A et B portaient des aimantations égales la déviation resterait nulle; la déviation $S_{\mathrm{c}}$ effectivement observée est due à la relaxation de l'aimantation nucléaire. Elle peut se traduire en première approximation en attribuant à la zone A une aimantation moyenne uniforme $M_{0}$ et à $\mathrm{B}$ une aimantation moyenne uniforme $k M_{0}$. On relève alors les déviations $S_{\mathrm{c}}$ en fonction du débit.

Par ailleurs, appelons $e_{\mathrm{A}}$ et $e_{\mathrm{B}}$ les déviations dues respectivement aux zones $\mathrm{A}$ et $\mathrm{B}$ portant une aimantation uniforme unité quand la cellule est en position de travail (face inférieure dans le plan de l'aimant central). Si $M_{0}^{\prime}$ est la valeur de l'aimantation calculée sans tenir compte du gradient d'aimantation le long de la cellule, on peut écrire :

$$
\begin{aligned}
& e_{\mathrm{A}} M_{0}^{\prime}+e_{\mathrm{B}} M_{0}^{\prime}=S \\
& e_{\mathrm{A}} M_{0}+k M_{0} e_{\mathrm{B}}=S \\
& e_{\mathrm{A}} M_{0}-k M_{0} e_{\mathrm{A}}=S_{\mathrm{c}}
\end{aligned}
$$

d'où l'on tire :

$$
\begin{aligned}
k & =\frac{e_{\mathrm{A}}\left(S-S_{\mathrm{c}}\right)}{e_{\mathrm{A}} S+e_{\mathrm{B}} S_{\mathrm{c}}} \\
M_{0} & =\frac{e_{\mathrm{A}} S+e_{\mathrm{B}} S_{\mathrm{c}}}{\left(e_{\mathrm{A}}+e_{\mathrm{B}}\right) e_{\mathrm{A}}} \\
\frac{M_{0}}{M_{0}^{\prime}} & =\frac{1}{k}\left(1-\frac{S_{\mathrm{c}}}{S}\right)=\frac{S-S_{\mathrm{c}}}{k S} .
\end{aligned}
$$

On a représenté, figure $5, M_{0}, M_{0}^{\prime}$ en fonction du débit pour une expérience particulière. La petitesse des corrections montre que l'approximation est suffisante et qu'il n'est pas nécessaire d'envisager une division en un plus grand nombre de zones.

3. RÉSUltats. - Les expériences, dont les résultats sont représentés figure 6 , ont été réalisées avec deux eaux de pureté différente. Le calcul de la susceptibilité effectué au point de débit optimal $d_{M}$ où la précision relative est la meilleure conduit à :

$$
\chi_{0}=\frac{M_{0}}{y_{\mathbf{M}} H_{\mathrm{p}}} \text {. }
$$

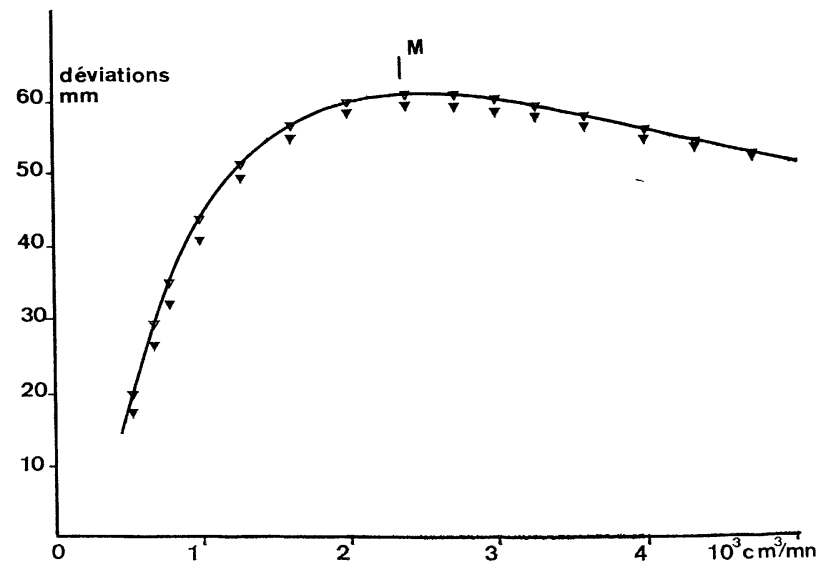

FIG. 5. - $\nabla$ : Points expérimentaux. $\nabla:$ Points expérimentaux corrigés. En traits pleins, la courbe théorique

$$
y=\exp \frac{-\alpha V_{\mathrm{r}}}{T_{1} d}\left(1-\exp \frac{-V_{\mathrm{r}}}{T_{1} d}\right)
$$

ajustée sur les points expérimentaux en faisant varier $\alpha$ et $T_{1}$ et en faisant se confondre les maximums par une affinité.

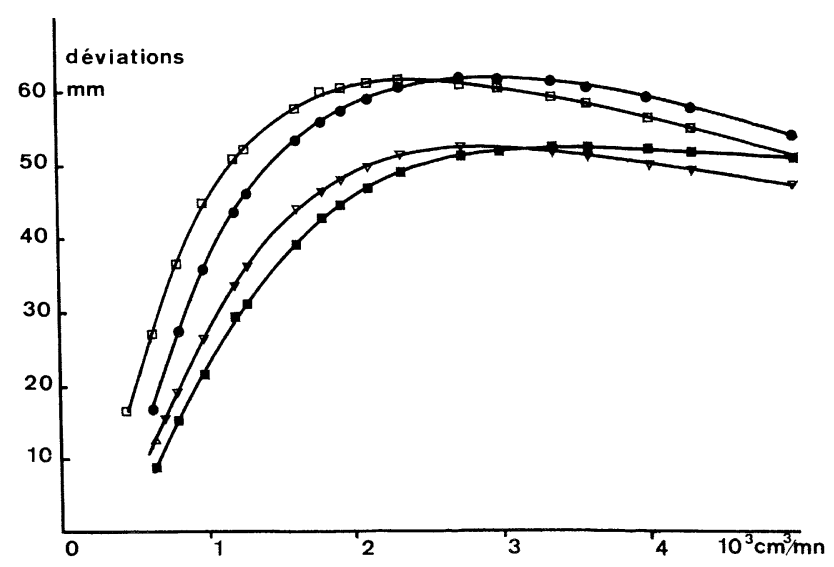

FIG. 6. - Résultats expérimentaux obtenus avec 2 eaux de pureté différente et deux valeurs du volume $V_{\mathrm{c}}$.

$$
\begin{array}{lrr}
\square & \alpha=0,20 & T_{1}=2,40 \mathrm{~s} \\
\nabla & 0,20 & 2,00 \mathrm{~s} \\
\nabla & 0,31 & 2,40 \mathrm{~s} \\
\square & 0,31 & 2,00 \mathrm{~s}
\end{array}
$$

On peut noter l'excellente coïncidence entre les courbes théoriques et les courbes expérimentales.

Les débits optimaux théoriques sont en bon accord avec l'expérience puisque l'on a :

$$
\begin{aligned}
& {\left[\begin{array}{rl}
\mathrm{d}_{\mathrm{M}} & =48 \mathrm{~cm}^{3} / \mathrm{s} \quad \text { et } d_{\text {Mthéorique }}=47,5 \mathrm{~cm}^{3} / \mathrm{s} \\
\alpha & =0,20 \\
T_{1} & =2,00 \mathrm{~s}
\end{array}\right.} \\
& {\left[\begin{array}{rl}
d_{\mathrm{M}} & =40 \mathrm{~cm}^{3} / \mathrm{s} \quad \text { et } \quad d_{\mathbf{M}^{\text {théorique }}}=39,6 \mathrm{~cm}^{3} / \mathrm{s} \\
\alpha & =0,20 \\
T_{1} & =2,40 \mathrm{~s}
\end{array}\right.} \\
& {\left[\begin{array}{rl}
d_{\mathrm{M}} & =48,5 \mathrm{~cm}^{3} / \mathrm{s} \text { et } \mathrm{d}_{\text {Mthéorique }}=49,2 \mathrm{~cm}^{3} / \mathrm{s} \\
\alpha & =0,31 \\
T_{1} & =2,40 \mathrm{~s}
\end{array}\right.}
\end{aligned}
$$


sachant que $H_{\mathrm{p}}=12.000$ gauss et en prenant pour valeur de la susceptibilité diamagnétique de l'eau $\chi=0,7216 \times 10^{-6}$ dans le système c. g. s., on trouve pour $\chi_{0}$ :

$$
\chi_{0}=3,22 \times 10^{-10} \text { dans le système C. G. S. }
$$

VII. Précision des résultats. — L'incertitude sur $\chi_{0}$ est de la forme :

$$
\frac{\Delta \chi_{0}}{\chi_{0}}=\frac{\Delta M_{0}}{M_{0}}+\frac{\Delta y_{\mathrm{m}}}{y_{\mathrm{m}}}+\frac{\Delta H_{\mathrm{p}}}{H_{\mathrm{p}}} .
$$

L'aimantation étant obtenue par comparaison avec le diamagnétisme (on fait en sorte que $S_{\text {diamagnétisme }} \simeq S_{\mathrm{max}}$ ), on peut écrire :

$$
\frac{\Delta M_{0}}{M_{0}} \leqslant 2\left(\frac{\Delta S}{S_{\max }}\right)
$$

D'autre part, le champ $H_{\mathrm{p}}$ est mesuré avec une précision de l'ordre de $0,5 \%$ en utilisant la linéarité de la loi champ-courant vérifiée sur le champ de fuite à l'aide d'un gaussmètre à haute sensibilité.

Précision sur y et sur $T_{1}$. - La stabilité de l'écoulement permet de déterminer le débit avec une précision supérieure à $0,5 \%$.

D'autre part :

$$
\Delta y=f^{\prime}(\alpha) \Delta+f^{\prime}\left(T_{1}\right) \Delta T_{1} .
$$

$\Delta \alpha$ et $\Delta T_{1}$ sont fonction de la précision $\Delta S$ des enregistrements et des écarts avec la courbe théorique. Leur détermination peut être faite par exemple en supposant que $\Delta \alpha$ et $\Delta T_{1}$ sont des erreurs indépendantes.

On obtient alors :

$$
\frac{\Delta y}{y}=\frac{\Delta S}{S}=\frac{V_{\mathrm{r}}}{T_{1} d} \Delta \alpha
$$

d'où

$$
\Delta \alpha=\frac{T_{1} d}{V_{\mathrm{r}}} \cdot \frac{\Delta S}{S}
$$

Par ailleurs

$$
\frac{\Delta y}{y}=\frac{\Delta S}{S}=\left[\frac{1}{\exp \left[\frac{V_{\mathrm{r}}}{T_{1} d}\right]-1}-\alpha\right] \frac{V_{\mathrm{r}}}{T_{1} d} \cdot \frac{\Delta T_{1}}{T_{1}}
$$

$\Delta T_{1}$ et $\Delta \alpha$ correspondent donc aux écarts qu'il faut faire subir à $\alpha$ et $T_{1}$ pour entraîner une variation $\Delta y / y$ supérieure à $\Delta S / S$.

On peut calculer $\Delta T_{1}$ et $\Delta \alpha$ pour chaque point de mesure. $\Delta S$ tel que $S \pm \Delta S / 2$ encadre la courbe théorique est dans tous les cas inférieur à $0,5 \mathrm{~mm}$. Nous avons reporté figure 7 l'enregistrement de la variation du champ magnétique créé sur le magnétomètre par l'inversion de l'aimantation nucléaire. La figure 8 représente les courbes donnant $\Delta \alpha$ et $\Delta T_{1}$ en fonction du débit $d$.

Notons que l'utilisation de débit compris entre A

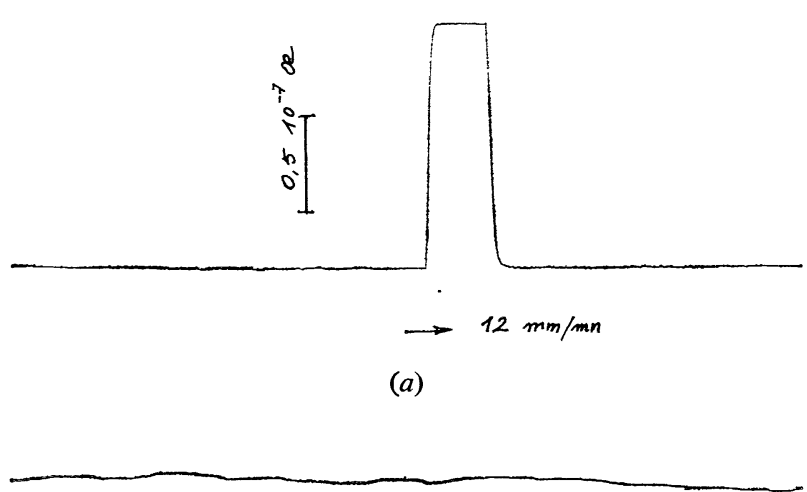

(b)

Fig. 7. - $-a$ ) Enregistrement en fonction du temps de la déviation du magnétomètre, lors d'un retournement. Une deuxième échelle verticale indique le champ produit au niveau du magnétomètre. $b$ ) Mise en évidence du bruit de signal après augmentation de la sensibilité.

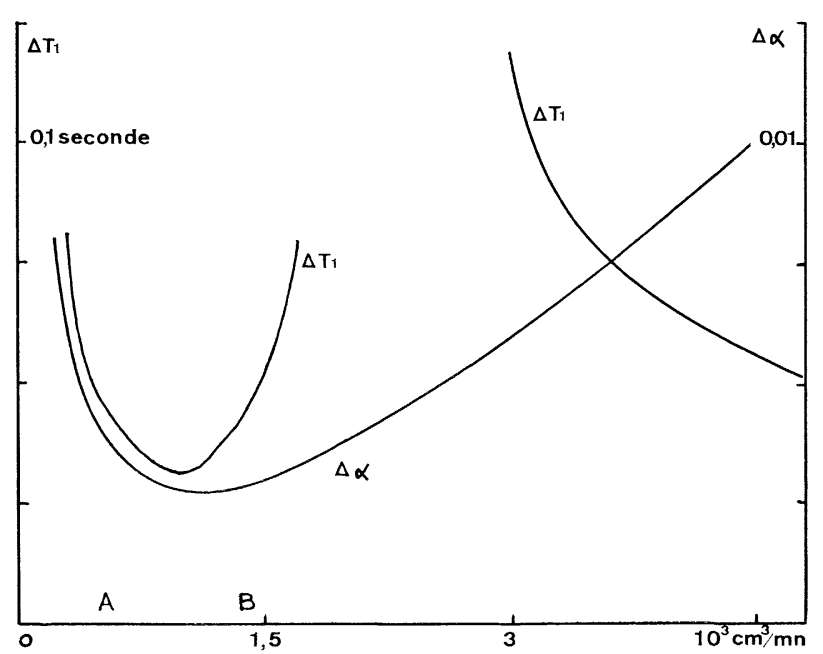

Fig. 8. - Valeurs, pour chaque point de mesure, de $\Delta T_{1}$ et $\Delta \alpha$, le calcul étant fait en considérant que $\Delta T_{1}$ et $\Delta \alpha$ sont des erreurs indépendantes.

et $\mathrm{B}$ dans une expérience de mesure de $T_{1}$, ou de $\alpha$, donne la plus grande précision, en particulier on a :

$$
\begin{aligned}
\Delta T_{1} & <0,05 \mathrm{~s} \\
\Delta \alpha & <0,005 .
\end{aligned}
$$

Précision sur $y_{\mathrm{m}}$. - Rappelons (I, 2) qu'au débit optimum $\Delta T_{1}$ ne joue aucun rôle, il vient donc :

$$
\frac{\Delta y_{\mathrm{m}}}{y_{\mathrm{m}}}=\frac{V_{\mathrm{r}}}{T_{1} d} \quad \Delta \alpha=\frac{\Delta S}{S_{\max }}
$$

soit

$$
\frac{\Delta y_{\mathrm{m}}}{y_{\mathrm{m}}} \leqslant 1 \%
$$

d'où

$$
\begin{aligned}
& \frac{\Delta \chi_{0}}{\chi_{0}} \leqslant 3 \% \\
& \chi_{0}=3,22 \pm 0,1 \times 10^{-10}
\end{aligned}
$$

dans le système u. e. m. c. g. s. 
Conclusion. - A notre connaissance, il s'agit de la deuxième mesure directe, à température ordinaire de la susceptibilité magnétique nucléaire statique d'un liquide, la première étant celle citée en référence [3] qui a donné $\chi_{0}=3,23 \times 10^{-10}$ dans le système c. g. $\mathrm{s}$. Les résultats obtenus sont en très bon accord avec le calcul théorique à partir de la valeur connue du moment magnétique du proton : $\chi_{0}=3,30 \times 10^{-10}$ dans le système c. g. s., pour l'eau à température de $19^{\circ} \mathrm{C}$.

Cette méthode permet de mesurer des concentrations de liquides en protons.

Par ailleurs la précision de $2 \%$ environ, obtenue sur les mesures de $T_{1}$ permet d'envisager d'autres mesures de temps de relaxation en champ faible et à température ordinaire. Citons à titre d'exemple l'étude de la corrosion d'un métal dans un liquide : un des signes de la corrosion d'un métal par un liquide est l'apparition dans le liquide d'ions de ce métal. Si ces ions sont paramagnétiques, leur apparition affecte fortement le temps de relaxation du liquide.

Remerciements. - Nous tenons à remercier MM. les Professeurs A. Kastler, E. Thellier et H. Benoit pour l'intérêt constant qu'ils ont porté à ce travail et les conseils qu'ils nous ont prodigués. Nous remercions également le Laboratoire de Catalyse Biologique pour sa collaboration dans le domaine de la pureté de l'eau.

\section{Bibliographie}

[1] Lazarew (B.) et Schubnikow (L.), Phys. Z. Sov., $1937,11,445$.

[2] Evans (D. F.), Phil. Mag., 1956, 8e série, 1, 370.

[3] Poulis (J. A.), Massen (C. H.) et Van Der Leeden (P.), Magn. and Elect., Resonance and relaxation, North Holland Publishing Co, 1963, 743.

[4] Bloch, Phys. Rev., 1964, 70, 460.

[5] Benort (H.), Thèse Ann. Physique, 1959, 4, 1439.
[6] Thellier (E.), Thèse, Paris, 1938.

[7] Pozzr (J. P.) et Thellier (E.), C. R. Acad. Sci., Paris, 1963, 257, 1037.

[8] Pozzi (J. P.), 1967, Thèse Docteur Ingénieur, Paris.

[9] Benoit (H.), François (M.), Pozzi (J. P.), Thellier (E.) et Kastler (A.), C. R. Acad. Sci., Paris, $1965,265,943-948$. 